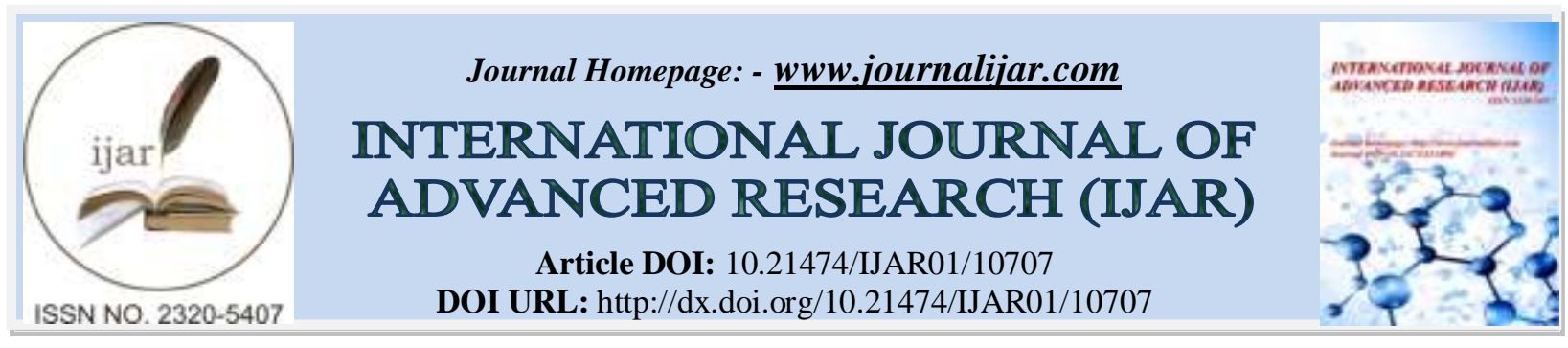

\title{
RESEARCH ARTICLE \\ SUCCESSFUL MANAGEMENT OF A CHALLENGING LONG STANDING LARGE SUPERFICIAL FEMORAL ARTERY PSEUDOANEURYSM
}

Dr. Vadivelu P, Dr. B. Kalavathy and Dr. M. Kamalakannan

Kovai Vascular Centre, Swathipolyclinic, Sowripalayam Rd, Coimbatore, Tamil Nadu-641028.

\section{Manuscript Info \\ Manuscript History \\ Received: 20 January 2020 \\ Final Accepted: 22 February 2020 \\ Published: March 2020}

Key words:-

Pseudoaneurysm, Penetrating Trauma

\begin{abstract}
Accidental penetrating trauma frequently involves the extremities, and can extend to involve their blood supply, causing exsanguinating hemorrhage and pseudoaneurysm in the involved blood vessel. Here we have a case of 27-year-old male presented with right lower extremity pain, swelling, and vascularCompromise following penetrating trauma which occurred 2 years back. Computed tomographic angiography revealed large distal superficial femoral artery pseudoaneurysm with distal popliteal artery occlusion. The patient underwent evacuation of pseudoaneurysm clots, excision with vein graft bypass and had a successful postoperative outcome. Our case illustrates importance of early identification and intraoperative challenges and its management of penetrating pseudoaneurysm when presented lately.
\end{abstract}

Copy Right, IJAR, 2020,. All rights reserved.

\section{Introduction:-}

Vascular injuries more common following penetrating trauma and these vascular injuries lead to devastating immediate complications like life threatening bleed, limb threatening ischemia or late complications like pseudoaneurysm formation. Extremity pseudoaneurysms are developed after penetrating trauma in $60 \%$ of arterial injuries. The diagnosis depends on the high rate of suspicion by the healthcare provider, and the longer the diagnosis takes, the more complications they may have, up to loss of the limb.[1-3]

\section{Case report:}

A 27year old male patient presented with swelling over right thigh for 2 years duration following penetrating trauma. Initially swelling for smaller and not had any treatment at that time(because resident of remote village). But for past one year it increased tremendously with pain over the swelling with lower limb intermittent claudication.

Corresponding Author:- Dr.Vadivelu P

Address:- Kovai Vascular Centre, Swathipolyclinic, Sowripalayam Rd, Coimbatore, Tamil Nadu641028. 

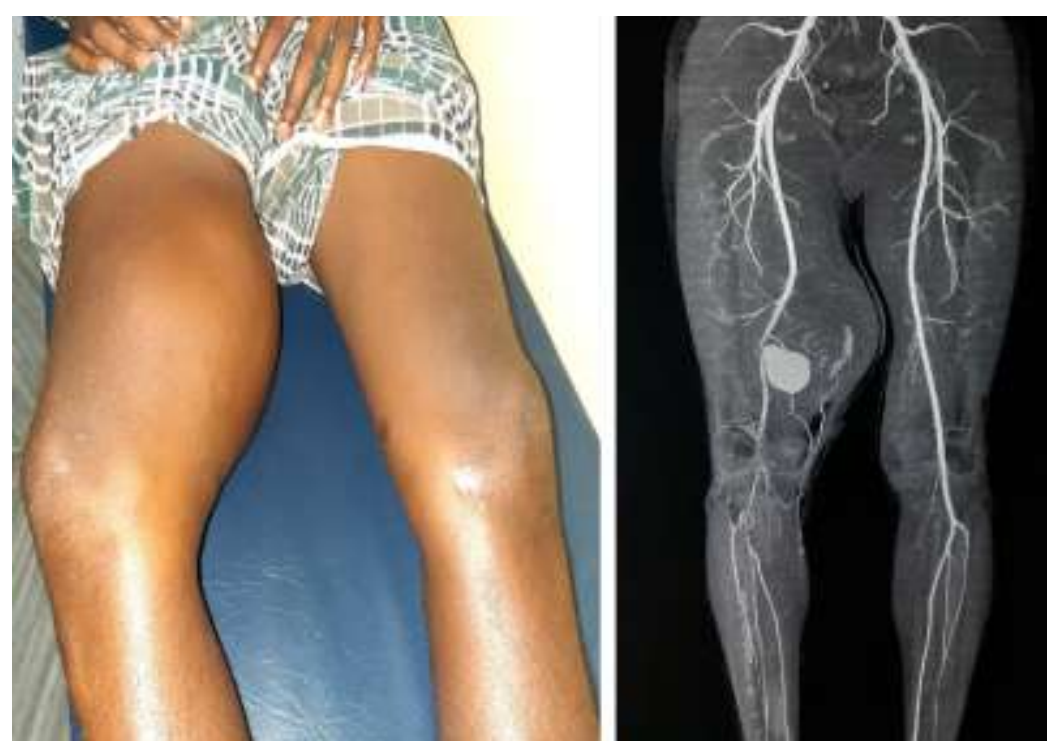

Figure 1:- Large pseudoaneurysm in medial aspect of right thigh.

On examination diffuse swelling of size $20 \times 20 \mathrm{~cm}$ seen in medial aspect of right thigh, swelling for firm, tense, tender, non pulsatile with skin over it stretched and shiny (Fig:1). Distal popliteal and tibial arteries pulses were absent. No distal neurological deficit seen. Patient on evaluation with CT angiogram showed large pseudoaneurysm arising from superficial femoral artery with surrounding clot measuring $16 \times 10 \mathrm{~cm}$ in size with distal popliteal artery occlusion. With diagnosis of pseudoaneurysm of superficial femoral artery with femoropopliteal occlusion, patient was planned for pseudoaneurysm repair with distal bypass.

\section{Procedure:}

Under epidural with spinal anesthesia, before exposing aneurysm sac, proximal common femoral/ superficial femoral arteries and distal popliteal arteries were exposed and controls taken. Longitudinal incision made over the medial aspect of thigh and incision deepened. Large Pseudoaneurysm sac arising from distal superficial femoral artery measuring $20 \times 20 \mathrm{~cm}$ was found with distorted tissue planes with dense adhesions due to prolonged compression of huge mass over surrounding structures. With clamping of both proximal and distal arteries, meticulously sac incised and opened with large amount of clots evacuated (around $1.2 \mathrm{~kg}$ ), thorough wash given and sac excised(Fig: 2). Sac was found adhering to femoral vein and surrounding structures, these were meticulously dissected. Clot send for culture sensitivity (found negative for microbes). Great saphenous vein harvested from opposite lower limb. Mid Superficial femoral artery to popliteal artery vein graft bypass done with 6'0 prolene. Good distal flow secured. Wound closed in layers with drain.Patient has two units of blood transfusion perioperatively even with above measures. Postoperative period uneventful and patient discharged after one week of hospital stay with no neurovascular deficit. 

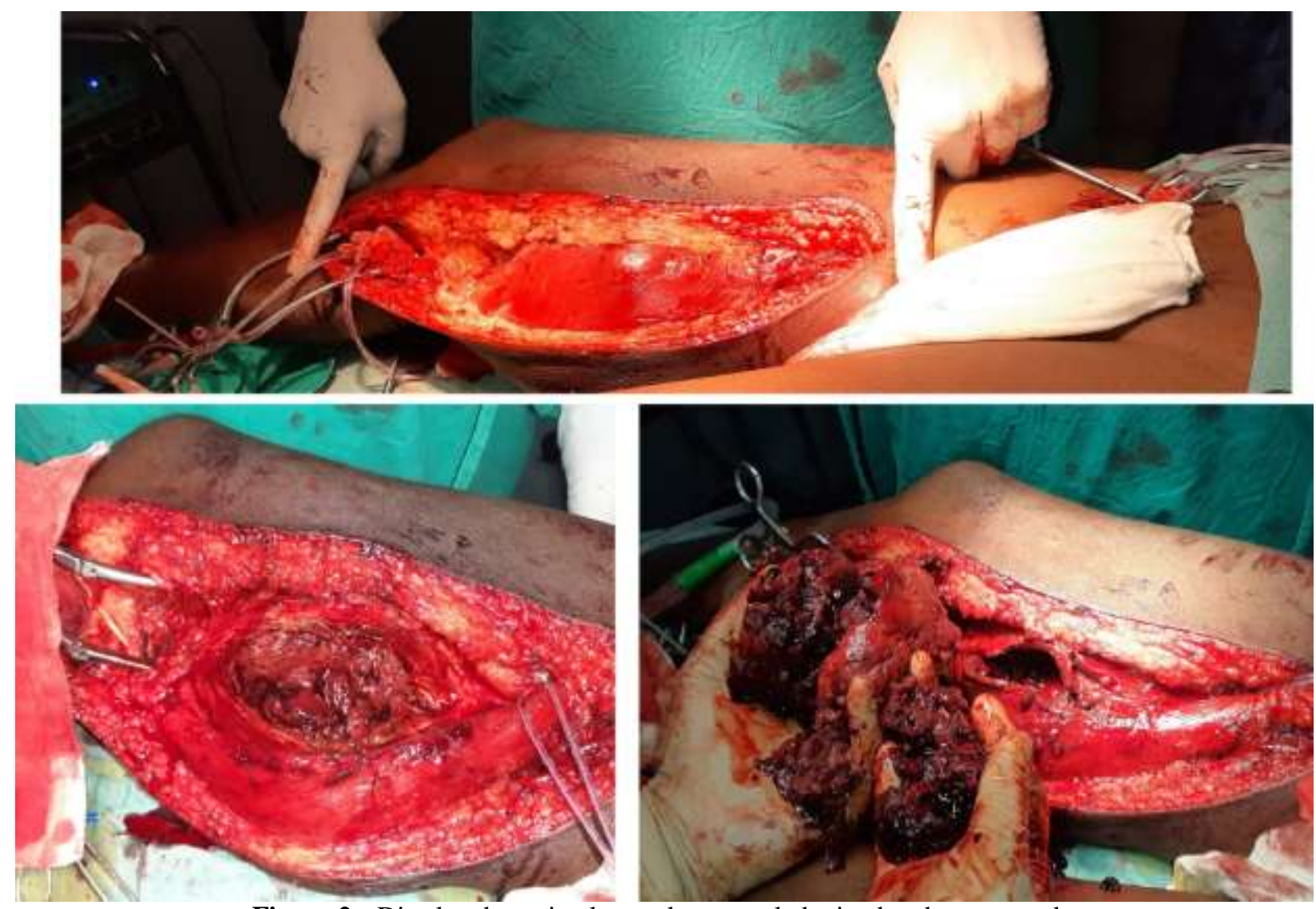

Figure 2:- Distal and proximal vascular control obtained and sac opened.

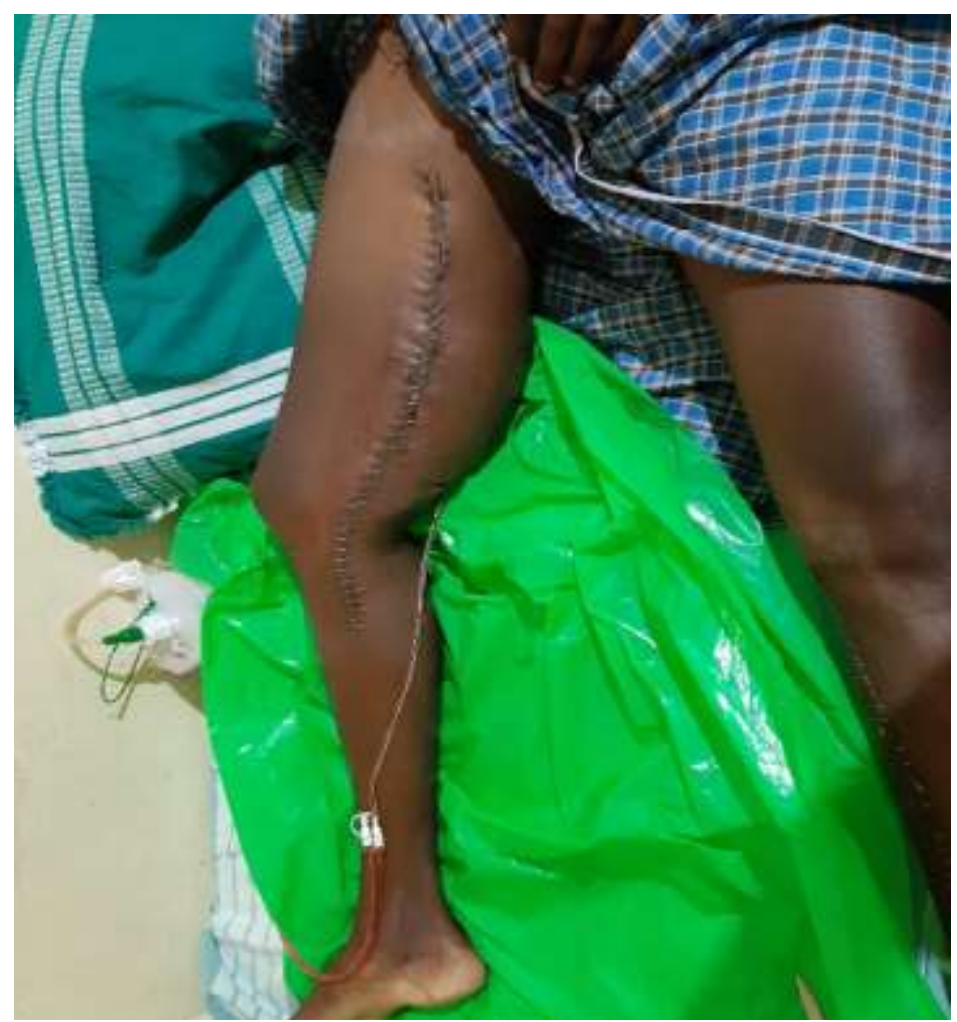

Figure 3:- postoperative. 


\section{Discussion:-}

Accidental trauma involving the extremities is becoming more common due to its connection with increasing social violence. The resultant arterial injuries can cause life-threatening bleeding, hematoma, distal ischemia due to thrombosis, laceration or disruption of the lumen, pseudoaneurysm formation or an arteriovenous fistula in involved segments. The detection and definition of vascular injury by angiography is essential for its optimal management [45]. A pseudoaneurysm is a contained arterial disruption involving the intimal and medial layers of the arterial wall. It can originate from a perforation by traumatic or iatrogenic injury, or can result from dehiscence of a surgical anastomosis. Pain is the most frequent complaint. Swelling, presence of a pulsatile mass, and paresthesia of the involved area can be variably present based on the location. [6,7]

Chronic traumatic aneurysm is developed by the partial or complete absorption of the periarterial hematoma at a fibrous sac level which is surrounded by the adjacent tissue. In general, the term "traumatic aneurysm" is utilized to refer to pseudoaneurysms, whether they are acute or chronic. Pseudoaneurysms must be treated as soon as possible, because of their risk of rupture or thrombosis. [7,8]

Thigh pseudoaneurysms are relatively rare, and usually result from direct injury to an arterial branch.Large sized pseudoaneurysm are common in distal superficial femoral artery, owing to deep seated location of in thigh.[4]These distal superficial femoral artery pseudoaneurysms are difficult to manage especially when presented late and as large ones. Because of deep seated location, no bony prominence for compression of bleed and artery course changes from medial to posterior in lower thigh along with distorted anatomy due to chronic nature and large mass displacing vital structures. In our case we faced all these challenges introperatively but with clear CT angiography images with thorough knowledge of lower limb anatomy with anticipation of above problems we were successful in managing this case.

\section{Conclusion:-}

Thus best way to manage distal superficial femoral artery pseudoaneurysm is early intervention and when presented late above challenges to be kept in mind for successful outcome.

\section{Acknowledgements:-}

The author would like to thank Ms.P.Asha (Staff nurse, Kovai Vascular Centre, Swathi polyclinic, Sowripalayam Rd, Coimbatore, Tamil Nadu 641028) and other Swathi polyclinic staff members for their constant support.

\section{References:-}

1. UçarHI,ÖçM,Doğan. Peripheral Vascular Injuries in CivilianPopulation. TurkiyeKlinikleri J CardiovascSci 2006;18:132-137.

2. Yetkin U, Bayrak S, Tetik B, et al. Surgical Approach to thePseudoaneurysms Of Lower extremity Arteries Developed afterGunshot Injuries. Journal of Thoracic \& Cardiovascular Surgery2007;10:2. Rutherford's vascular surgery, $9^{\text {th }}$ edition.

3. M. A. Gómez-Álvarez a, G. E. Muñoz-Maldonado et al: Surgical treatment of a pseudoaneurysm of the femoral arterysecondary to a gunshot wound. Clinical case report.MedicinaUniversitaria 2014;16(64):133-135

4. Frykberg ER, Crump JM, Vines FS, et al (1989) A reassessment of the role of arteriography in penetrating proximity extremity trauma: a prospective study. J Trauma 29: 1041-1052

5. S.Sharma ,B.Bhargava , M.Mahapatra , R.Malhotra et al :Pseudoaneurysm of the superficial femoral artery following accidental trauma: result of treatment by percutaneous stent-graftplacement. Eur. Radiol. 9, 422-424 (1999)

6. Schwartz LB, Clark ET, Gewertz BL. Anastomotic and otherpseudoaneurysms. In: Rutherford RB, ed. Vascular Surgery. 5 thed. Philadelphia: WB Saunders; 2000:752-763.

7. StefanoSchena, CharlesAOwenset al:Delayed Presentation of a Posttraumatic Superficial

8. Femoral Artery Pseudoaneurysm. American College of SurgeonsVol. 203, No. 2, August 2006:250-251

9. Rutherford RB. Diagnostic evaluation of extremity vascular injuries. SurgClin North Am 1988;68:683-691. 